\title{
Immune inflammation indicators in anal cancer patients treated with concurrent chemoradiation: training and validation cohort with online calculator (ARC: Anal Cancer Response Classifier) [Corrigendum]
}

\author{
Casadei-Gardini A, Montagnani F, Casadei C, et al. \\ Cancer Manag Res. 2019;11:3631-3642.
}

The authors have advised that the affiliations are not correct in the published paper (page 3631). They have provided revised author and affiliations lists, as follows, and confirm that the modifications do not alter their conflicts of interest status in regard to this work.

Andrea Casadei-Gardini, ${ }^{1,2}$ Francesco Montagnani, ${ }^{3}$ Chiara Casadei, ${ }^{1}$ Francesca Arcadipane, ${ }^{4}$ Kalliopi Andrikou, ${ }^{2}$ Deborah Aloi, ${ }^{5}$ Alessandra Anna Prete, ${ }^{6}$ Maria Giulia Zampino, ${ }^{7}$ Antonella Argentiero, ${ }^{8}$ Giuseppe Pugliese, ${ }^{2}$ Stefania Martini, ${ }^{4}$ Giuseppe Carlo Iorio, ${ }^{4}$ Mario Scartozzi, ${ }^{9}$ Massimiliano Mistrangelo, ${ }^{10}$ Lorenzo Fornaro, ${ }^{11}$ Paola Cassoni, ${ }^{12}$ Giorgia Marisi, ${ }^{13}$ Veronica Dell'Acqua, ${ }^{14}$ Paola Simona Ravenda, ${ }^{7}$ Sara Lonardi, ${ }^{6}$ Nicola Silvestris, ${ }^{8}$ Berardino De Bari, ${ }^{5}$ Umberto Ricardi, Stefano Cascinu, ${ }^{2}$ Pierfrancesco Franco ${ }^{4}$

${ }^{1}$ Department of Medical Oncology, Istituto Scientifico Romagnolo per lo Studio e la Cura dei Tumori (IRST) IRCCS, Meldola, Italy; ${ }^{2}$ Department of Hematology and
Oncology, University of Modena and Reggio Emilia, Modena, Italy; ${ }^{3}$ Oncology Department, SOC Oncology, ASL (Health Local Authority), Biella, Italy; ${ }^{4}$ Department of Oncology, Radiation Oncology, University of Turin, Turin, Italy; ${ }^{5}$ Radiation Oncology Department, Centre Hospitalier Régional Universitaire "Jean Minjoz", Besançon Cedex, France; ${ }^{6}$ Unit of Medical Oncology 1, Department of Clinical and Experimental Oncology, Istituto Oncologico Veneto, IRCCS, Padua, Italy; ${ }^{7}$ Division of Gastrointestinal Medical Oncology and Neuroendocrine Tumors, European Institute of Oncology, IRCCS, Milan, Italy; ${ }^{8}$ Medical Oncology Unit, Cancer Institute Giovanni Paolo II, Bari, Italy; ${ }^{9}$ Department of Medical Oncology, University of Cagliari, Cagliari, Italy;

${ }^{10}$ Department of Surgical Sciences, University of Turin, Turin, Italy; ${ }^{11}$ Unit of Medical Oncology 2, Azienda Ospedaliero-Universitaria Pisana University, Pisa, Italy; ${ }^{12}$ Department of Medical Sciences, Pathology Unit, University of Turin, Turin, Italy; ${ }^{13}$ Biosciences Laboratory, Istituto Scientifico Romagnolo per Lo Studio e La Cura Dei Tumori (IRST) IRCCS, Meldola, Italy; ${ }^{14}$ Department of Radiotherapy, IRCCS, European Institute of Oncology, Milan, Italy
Cancer Management and Research

Publish your work in this journal

Cancer Management and Research is an international, peer-reviewed open access journal focusing on cancer research and the optimal use of preventative and integrated treatment interventions to achieve improved outcomes, enhanced survival and quality of life for the cancer patient.
The manuscript management system is completely online and includes a very quick and fair peer-review system, which is all easy to use. Visit http://www.dovepress.com/testimonials.php to read real quotes from published authors. 